\title{
Liquid-Vapour Phase Change Rates and Interfacial Entropy Production
}

\author{
C.A. Ward \\ Department of Mechanical and Industrial Engineering, University of Toronto, Toronto, \\ Canada \\ Communicated by W. Rudzinski, Lublin, Poland
}

Registration Number 948

\begin{abstract}
Measurements have recently been made of the thermodynamic conditions at the interface during steady state, liquid-vapour phase transitions. In these stationary, nonequilibrium states discontinuities were recorded in the temperature and other intensive properties. Independently of whether evaporation or condensation was taking place, it was found that the interfacial temperature was higher in the vapour phase than that in the liquid. The expression for the interfacial entropy production during these phase change processes is formulated using statistical rate theory. The interfacial entropy production rate is not a minimum when there is a phase change process taking place, but if the system is required to be closed (no net phase change), the interfacial entropy production rate is a minimum. States of minimum entropy production rate can exist arbitrarily far from equilibrium, provided a certain relation exists between the properties of the substance undergoing the phase change. For water, it is found that states of minimum entropy production rate are only slightly displaced from the stationary, nonequilibrium states existing when a net phase change rate is present.
\end{abstract}

\section{Introduction}

If an open, two-phase system were maintained in a steady state with discontinuities in the intensive properties across the phase boundary, one would, in general, expect an entropy production at the interface between the two phases. The interfacial conditions during the steady state evaporation and condensation of water [1-4] and the evaporation of methylcyclohexane and of octane [5] each held in such a state were recently measured. Independently of the direction of net molecular transport at the interface, the interfacial temperature was found to be higher in the vapour than in the liquid. The largest temperature discontinuity was found during steady state water 
evaporation and was $7.8^{\circ} \mathrm{C}$. The interfacial temperature in each phase was the lowest temperature in that phase. Thermal energy was transported from within each phase to the interface. This energy went into the latent heat associated with the net phase change rate [3].

Herein, the expression for the interfacial entropy production rate, $\dot{\sigma}_{L V}$ is formulated using statistical rate theory (SRT) [1-21]. This theory is based on conventional (Copenhagen) quantum mechanics (QM) and a hypothesis that claims the rate of exchange between QM states of an isolated system has the same value. Although the conventional quantum mechanics approach for predicting irreversibility has been questioned by some [22], for an isolated system SRT predicts an irreversible evolution to an equilibrium configuration that corresponds to the maximum number of QM states [1]. The approach has been examined in a number of nonequilibrium circumstances, including unsteady evaporation [2], gas adsorption kinetics on homogeneous and heterogeneous surfaces [7-14], surface diffusion [15], membrane transport [16], electron transfer reactions [17], crystal dissolution [18] and gas absorption by liquids [19-21]. In each case, the SRT procedure received support from the comparison with experimental measurements, but it received particularly strong support from the comparison with measurements of the predicted phase change rate of water [1-4], thermal desorption of $\mathrm{CO}$ from $\mathrm{Ni}(111)$ [11] and gas absorption by liquids [19-21]. In these latter cases, predictions could be made of the kinetics in which there were no fitting parameters, and the results compared with measurements.

Using the SRT approach, we show that the stationary, nonequilibrium states of open systems associated with the liquid-vapour phase transition are characterized by the change in entropy that results from one molecule transferring from the liquid to the vapour phase, $\Delta s_{L V}$ [1-5]. The sign of this quantity determines the direction of the net molecular transport at the interface, i.e., whether there is evaporation or condensation taking place [1], and when $\Delta s_{L V}$ is zero the rate of entropy production is a minimum. When used with SRT, the conditions at the interface when a phase change process is taking place at a particular rate can be predicted and compared with measurements. When there is a stationary, net phase change rate, we find that $\dot{\sigma}_{L V}$ is not a minimum. Requiring $\dot{\sigma}_{L V}$ to be a minimum is tantamount to requiring the net phase change rate to be zero. Such states can exist arbitrarily far from equilibrium provided a special relation exists between the properties of the substance considered. We show that for water, states of minimum entropy production rate could easily exist in which large discontinuities in the intensive properties would be present across the phase boundary.

\section{Statistical rate theory expression for the net evaporation flux and entropy production rate}

Suppose that at one instant, a volume liquid phase is in contact with a small volume of its vapour. The system will be assumed to be partitioned into three phases - the substrate liquid, the surface phase and vapour phase. The substrate liquid, surface and vapour form an isolated system. Each portion of the system is assumed to be in local equilibrium, but disequilibrium exists between the surface phase and the vapour 
phase. If the instantaneous values of the temperatures and pressures in the liquid and vapour phases, $T^{L}, T^{V}, P^{L}$ and $P^{V}$, are known, as well as the molecular and material properties of the substance changing phase, the objective of SRT is to predict the evaporation flux at that instant. We suppose that the surface is spherical and of radius $R_{0}$, and that the pressures in the respective phases are related to the curvature through the Laplace relation:

$$
P^{L}-P^{V}=\frac{2 \gamma^{L V}}{R_{0}}
$$

where $\gamma^{L V}$ is the surface tension.

At the instant considered, the change in entropy resulting from one molecule transferring from the surface to the vapour phase is denoted as $\Delta s_{\sigma V}$. If the chemical potentials are denoted as $\mu^{\sigma}, \mu^{V}$, and the enthalpy in the volatile phase as $h^{V}$, the Euler relation may be used to express the total entropy of the vapour

$$
S^{V}=N^{V}\left(\frac{h^{V}-\mu^{V}}{T^{V}}\right)
$$

and that of the surface phase as

$$
S^{\sigma}=N^{\sigma}\left(\frac{u^{\sigma}-\mu^{\sigma}}{T^{\sigma}}\right)-\frac{\gamma A}{T^{\sigma}}
$$

where the intensive internal energy of the surface phase is denoted as $u^{\sigma}$, its surface tension as $\gamma$, its area as $A$ and its temperature as $T^{\sigma}$. When there are $N^{\sigma}$ molecules in the surface phase and $N^{V}$ in the vapour phase, the total entropy of the isolated system may be written

$$
S\left(N^{V}, N^{\sigma}\right)=N^{\sigma}\left(\frac{u^{\sigma}-\mu^{\sigma}}{T^{\sigma}}\right)-\frac{\gamma A}{T^{\sigma}}+N^{V}\left(\frac{h^{V}-\mu^{V}}{T^{V}}\right)+S^{L}\left(N^{V}, N^{\sigma}\right)
$$

where $S^{L}\left(N^{V}, N^{\sigma}\right)$ is the entropy of the substrate liquid at the instant considered, $A$ is the surface area. Now suppose a molecule is transferred from the surface phase to the vapour. Since the system is isolated, in this transition there must be both conservation of molecular number and of energy. Also, we assume the intensive properties are unchanged as a result of this transition. Then the change in entropy of the isolated system, $\Delta s_{\sigma V}$ may be written

$$
\Delta s_{\sigma V}=\left(\frac{h^{V}-\mu^{V}}{T^{V}}\right)+\left(\frac{\mu^{\sigma}-u^{\sigma}}{T^{\sigma}}\right)+S^{L}\left(N^{V}+1, N^{\sigma}-1\right)-S^{L}\left(N^{V}, N^{\sigma}\right)
$$

and conservation of energy gives

$$
\begin{aligned}
N^{V} h^{V}+N^{\sigma} u^{\sigma}+U^{L}\left(N^{V}, N^{\sigma}\right)= & \left(N^{V}+1\right) h^{V}+\left(N^{\sigma}-1\right) u^{\sigma} \\
& +U^{L}\left(N^{V}+1, N^{\sigma}-1\right)
\end{aligned}
$$


or

$$
U^{L}\left(N^{V}+1, N^{\sigma}-1\right)-U^{L}\left(N^{V}, N^{\sigma}\right)=u^{\sigma}-h^{V}
$$

We approximate the substrate liquid as a reservoir for this transition, then

$$
\Delta s_{\sigma V}=\left(\frac{h^{V}-\mu^{V}}{T^{V}}\right)+\left(\frac{\mu^{\sigma}-u^{\sigma}}{T^{\sigma}}\right)+\frac{u^{\sigma}-h^{V}}{T^{L}}
$$

We neglect any difference between the temperatures and the chemical potentials of the substrate liquid and those of the surface phase. The change in entropy resulting from one molecule transferring from the liquid to the vapour phase, now denoted as $\Delta s_{L V}$ becomes

$$
\Delta s_{L V}=\left(\frac{\mu^{L}}{T^{L}}-\frac{\mu^{V}}{T^{V}}\right)+h^{V}\left(\frac{1}{T^{V}}-\frac{1}{T^{L}}\right)
$$

We note that the expression for $\Delta s_{L V}$ given in Eq. (8) is the same as that obtained when the molecule was assumed to transfer directly from the liquid phase to the vapour phase [4]. With the approximations made, Eq. (3) becomes

$$
S^{\sigma}=N^{\sigma}\left(\frac{u^{\sigma}-\mu^{L}}{T^{L}}\right)-\frac{\gamma A}{T^{L}}
$$

As may be seen by comparing Eq. (9) with Eq. (2), even with these approximations, the two expressions for the entropy are different, but the expression for the change in entropy that results from one molecule transferring from one phase to the other phase is the same.

From statistical mechanics, an approximate expression for $\Delta s_{L V}$ may be obtained [4]

$$
\begin{aligned}
\frac{\Delta s_{L V}}{k}= & 4\left(1-\frac{T^{V}}{T^{L}}\right)+\left(\frac{1}{T^{V}}-\frac{1}{T^{L}}\right) \sum_{l=1}^{3}\left(\frac{\hbar \omega_{l}}{2 k}+\frac{\hbar \omega_{l} / k}{\exp \left(h \omega_{l} / k T^{V}\right)-1}\right) \\
& +\frac{v_{\text {sat }}^{L}}{k T^{L}}\left[P^{L}-P_{\text {sat }}\left(T^{L}\right)\right]+\ln \left[\left(\frac{T^{V}}{T^{L}}\right)^{4}\left[\frac{P_{s a t}\left(T^{L}\right)}{P^{V}}\right]\right]+\ln \left[\frac{q_{v i b}\left(T^{V}\right)}{q_{v i b}\left(T^{L}\right)}\right],
\end{aligned}
$$

where, $q_{v i b}$ is the vibrational partition function:

$$
q_{v i b}=\prod_{l=1}^{3} \frac{\exp \left(-\hbar \omega_{l} /(2 k T)\right)}{1-\exp \left(-\hbar \omega_{l} / k T\right)}
$$

For the water molecule, the three measured vibrational frequencies have values of 1590,3651 and $3756 \mathrm{~cm}^{-1}$ [23]. 
If it is assumed that in the isolated system: i) the rate of transition between quantum states is the same for all the available states of the isolated system, i.e., all the states that are within the energy uncertainty, and ii) the Boltzmann definition of entropy is valid, then the expression for the instantaneous, unidirectional evaporation flux, $\tau_{L V}$ that is obtained from the transition probability concept of quantum mechanics may be written [4]

$$
\tau_{L V}=\frac{A_{0}\left|V_{\nu \varepsilon}\right|^{2} \zeta_{\Delta E}}{\hbar}\left(\exp \left[\frac{\Delta s_{L V}}{k}\right]\right)
$$

where $\left|V_{\nu \varepsilon}\right|^{2}$ is the matrix element corresponding to a transition between quantum mechanical (QM) states of different molecular distributions, $\zeta_{\Delta E}$ is the QM state density which has been assumed uniform over the range of quantum states that are within the energy uncertainty of the isolated system, and $A_{0}$ is the value of the integral

$$
A_{0}=\int_{-\pi / 2}^{\pi / 2} \frac{\sin (y)}{y} d y
$$

and $k$ is the Boltzmann constant. Note that according to the assumptions made, $\left|V_{\nu \varepsilon}\right|^{2} \zeta_{\Delta E}$ is a constant for the isolated system. Similarly, one finds that the unidirectional condensation flux may be written

$$
\tau_{V L}=\frac{A_{0}\left|V_{\nu \varepsilon}\right|^{2} \zeta_{\Delta E}}{\hbar}\left(\exp \left[\frac{-\Delta s_{L V}}{k}\right]\right)
$$

Since $\Delta s_{L V}$ vanishes under equilibrium conditions, the values of $\tau_{V L}$ and $\tau_{L V}$ are equal at this condition. Each has the value $\left(A_{0}\left|V_{\nu \varepsilon}\right|^{2} \zeta_{\Delta E}\right) / \hbar$ and this quantity has been interpreted as the equilibrium exchange rate between the phases [1-4]. This provides an enormous simplification because the integrals appearing in $\left|V_{\nu \varepsilon}\right|^{2}$ do not have to be performed. Rather, an approximate expression for the equilibrium exchange rate between the phases may be determined from a physical model. For example, if the vapour phase is approximated as an ideal gas and it is assumed that each molecule from the vapour phase that strikes the liquid-vapour interface condenses, the equilibrium exchange rate between the phases would be equal to the collision frequency of the vapour phase molecules with the liquid [1, 4] then:

$$
\frac{A_{0}\left|V_{\nu \varepsilon}\right|^{2} \zeta_{\Delta E}}{\hbar}=\frac{P_{e}^{V}}{\sqrt{2 \pi m k T_{e}}}
$$

where the equilibrium temperature and pressure in the isolated system are denoted as $T_{e}, P_{e}^{V}$, and $m$ denotes the mass of the molecules transported between phases. The values of $T_{e}, P_{e}$ would depend on the constraints existing on the isolated system, but the values of these equilibrium properties are determined by the values of the total energy, volume and mass of the isolated system using the entropy-maximum principle. 
The net evaporation flux $j_{L V}$ is the difference between $\tau_{L V}$ and $\tau_{V L}$. From Eqs. (12) and (13), one finds

$$
j_{L V}=2\left(\frac{A_{0}\left|V_{\nu \varepsilon}\right|^{2} \zeta_{\Delta E}}{\hbar}\right) \sinh \left(\frac{\Delta s_{L V}}{k}\right)
$$

Note that the expression for the unidirectional evaporation flux depends on both the chemical potentials and the temperatures in the liquid and vapour phases. Thus, unlike the assumption made in classical kinetic theory [24], the rate at which the liquid is predicted to evaporate depends on the conditions in both the liquid and vapour phases.

The interfacial entropy production rate, $\dot{\sigma}_{L V}$ is given by the product of $j_{L V} \Delta s_{L V}$ or

$$
\dot{\sigma}_{L V}=2 \Delta s_{L V}\left(\frac{A_{0}\left|V_{\nu \varepsilon}\right|^{2} \zeta_{\Delta E}}{\hbar}\right) \sinh \left(\frac{\Delta s_{L V}}{k}\right)
$$

This expression for $\dot{\sigma}_{L V}$ is never negative. It has a minimum when $\Delta s_{L V}$ is zero and its value at the minimum is zero. A necessary condition for equilibrium across the phase boundary is that $\Delta s_{L V}$ vanishes, but it is not a sufficient condition.

\section{States of minimum interfacial entropy production rate}

In order for the rate of interfacial entropy production to be a minimum, it is only necessary that $\Delta s_{L V}$ is zero. This condition requires that a special relation exists between the properties in the liquid and vapour phases. We first establish these conditions.

The expression for $\Delta s_{L V}$ is given in Eq. (8). If one combines the Euler relation in each phase:

$$
\mu^{j}=h^{j}-T^{j} s^{j} \quad j=L \text { or } V
$$

with the expression for $\Delta s_{L V}$, then one finds

$$
\Delta s_{L V}=\left(s^{V}-s^{L}\right)-\frac{1}{T^{L}}\left(h^{V}-h^{L}\right)
$$

Hence, in order for $\Delta s_{L V}$ to vanish

$$
\left(s^{V}-s^{L}\right)=\frac{1}{T^{L}}\left(h^{V}-h^{L}\right)
$$

Physically, this result indicates $\Delta s_{L V}$ vanishes when the entropy increase during evaporation (or decrease during condensation) that results from the change in phase $\left(s^{V}-s^{L}\right)$ is balanced by the entropy decrease during evaporation (or increase during condensation) of the liquid phase due to the transfer of energy $\left(h^{V}-h^{L}\right)$. 
As will be seen, the interfacial conditions that are required to predict the evaporation flux at one instant in an isolated system are the interfacial radius $R_{0}$, the interfacial temperatures in each phase $T^{L}, T^{V}$ and the pressure in the vapour phase, $P^{V}$. We suppose that if the same interfacial conditions existed at one instant in the isolated system and in a system operating in steady state, then at that instant, the phase change flux, $j_{L V}$ would be the same in the two systems $[1,4]$. The future in the two systems would be totally different. The isolated system would evolve to a final equilibrium state, but the steady state system could remain unchanged.

If Eq. (19) were satisfied in an isolated system at one instant, the system could not be maintained in that state because neither energy nor mass could be supplied to maintain that state. However, in principle such a state could be maintained in an open system, since both energy and mass could be supplied (or withdrawn) to (from) such a system.

\subsection{Calculations of the interfacial entropy production rate}

We assume that the liquid-phase volume in the isolated system would be sufficient so that the final, equilibrium temperature in the isolated system would be approximately the same as the instantaneous interfacial temperature, $T^{L}$ of the liquid phase:

$$
T_{e}=T^{L}
$$

and that when the isolated system has evolved to equilibrium, the radius of the liquidvapour interface will be approximately equal to the instantaneous value, $R_{0}$. Under equilibrium conditions, the chemical potentials in the liquid and vapour phases must be equal.

If the liquid phase is approximated as incompressible and the vapour phase as an ideal gas, then the equality of the chemical potentials allows the pressure in the vapour phase to be expressed in terms of properties in the liquid phase [4]:

$$
P_{e}^{V}=P_{\text {sat }}\left(T^{L}\right) \exp \left[\frac{v_{\text {sat }}^{L}}{k T}\left[P_{e}^{L}-P_{\text {sat }}\left(T^{L}\right)\right]\right]
$$

and when this expression is combined with Eq. (1), one finds

$$
P_{e}^{L}=P_{\text {sat }}\left(T^{L}\right) \exp \left[\frac{v_{\text {sat }}^{L}}{k T^{L}}\left[P_{e}^{L}-P_{\text {sat }}\left(T^{L}\right)\right]\right]+\frac{2 \gamma^{L V}\left(T_{L}\right)}{R_{0}}
$$

For given values of the instantaneous properties $T^{L}, R_{0}$ the value of $P_{e}^{L}$ may now be calculated iteratively from Eq. (22). The value of $P_{e}^{V}$ may then be calculated from Eq. (21), and the value of $\left(A_{0}\left|V_{\nu \varepsilon}\right|^{2} \zeta_{\Delta E} / \hbar\right)$ from Eq. (14). Thus, the measured values of $T^{L}, R_{0}$ allow one to determine the equilibrium exchange rate between the liquid and vapour phases of the isolated system. 


\section{Steady state evaporation or condensation of water}

We now compare the conditions existing at the interface when water undergoes a steady state phase change with the conditions that would exist if the interfacial entropy production rate were a minimum. The measured conditions in the liquid and vapour phases during steady state evaporation and steady state condensation of water were reported in Ref. [1] and are listed in Table 1.

\subsection{Steady state evaporation}

Briefly, the experimental procedure was to maintain water at the mouth of a small funnel. The pressure above the funnel was reduced to produce evaporation, and simultaneously water was pumped into the bottom of the funnel at a rate equal to the total evaporation rate, $J_{L V}$ of the water at the mouth of the funnel. Hence, the liquidvapour interface position was maintained constant. The value of $J_{L V}$ was measured in each experiment. The mouth opening of the funnel was $7 \mathrm{~mm}$ and the height of the liquid-vapour interface above the funnel mouth was $\sim 1 \mathrm{~mm}$ in each experiment. As a result, the interface was approximately spherical. The conditions measured on the centreline included the interfacial radius $R_{0}$, and the interfacial temperatures in each phase $T^{L}, T^{V}$. The pressure in the vapour phase, $P^{V}$ a few centimeters above the interface, was also measured. The average evaporation flux, $j_{L V}$ was then determined from these measurements.

\subsection{Steady state condensation}

In the condensation experiments of Ref. [1], the annular region between two concentric cylinders was partially filled with water. The funnel with water at the mouth was on the centreline of the cylinders. The water in the annular region was heated and the water in the funnel was cooled. This produced condensation at the liquid-vapour interface that was maintained at the mouth of the funnel. Water was withdrawn from the bottom of the funnel at a rate that kept the position of the liquidvapour interface at the funnel mouth constant. Thus, the total rate of condensation could be measured by measuring the rate at which the liquid was withdrawn. The conditions on the centreline that were measured included the interfacial radius $R_{0}$, the interfacial temperatures in each phase $T^{L}, T^{V}$ and the pressure in the vapour phase, $P^{V}$. Since the interface curvature was approximately spherical, the average condensation flux, $j_{V L}$ could be determined from the measurements. (Note that the condensation flux is a negative evaporation flux, $j_{V L}=-j_{L V}$.)

Since the instantaneous pressure in the vapour phase is the variable that can be measured with the least accuracy, the procedure adopted has been to predict the pressure in the vapour phase at which a given evaporation flux, $j_{L V}$ would occur and then to compare the measured pressure in the vapour with that predicted [1-5]. Thus, to predict the pressure in the vapour phase, $P^{V}$ one inserts the measured values of $j_{L V}, T^{V}, T^{L}$, and $R_{0}$ into Eq. (15) and iterates on $P^{V}$ until the equation is satisfied. The values of the pressure in the vapour phase calculated by this procedure, denoted as $P_{R}$ are listed in Table 1 . Note that the predicted value of the pressure is not 


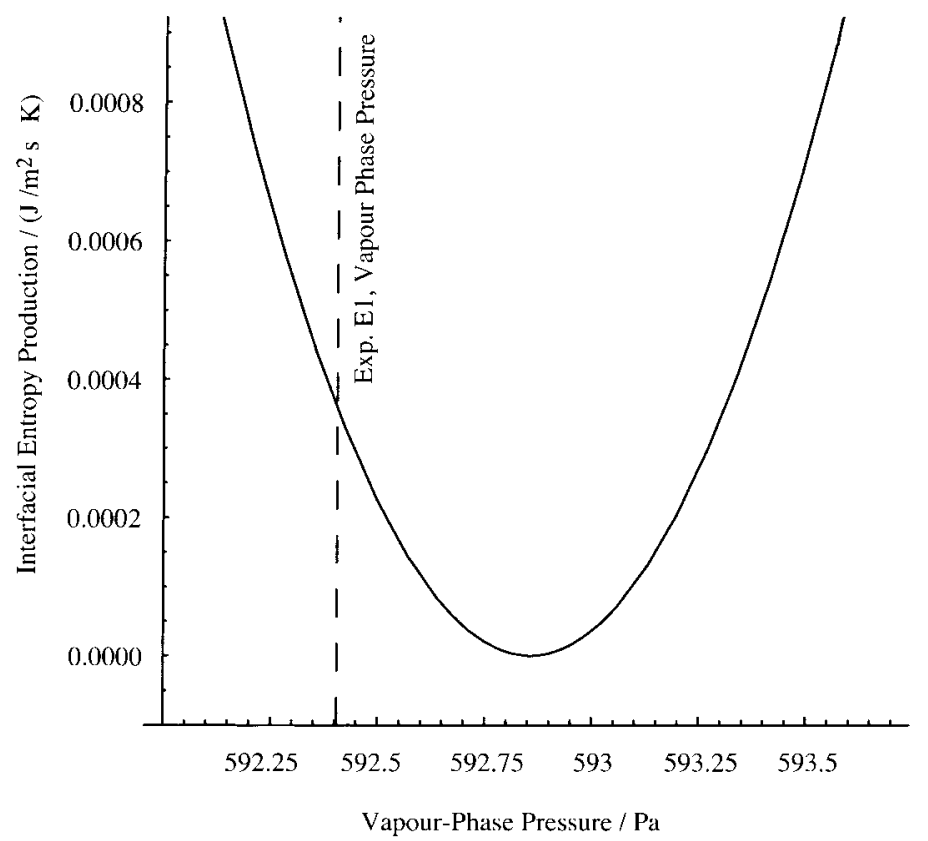

Fig. 1. Interfacial entropy production during steady state evaporation as a function of the vapour-phase pressure. The calculations were made for the values of $T^{L}, T^{V}$ and $R_{0}$ of evaporation experiment E1 (Table 1). The measured evaporation rate was $1.017 \mathrm{~g} / \mathrm{m}^{2} \mathrm{~s}$ and the predicted pressure was $592.404 \mathrm{~Pa}$. If the steady state system were operating at the minimum rate of interfacial entropy production, the vapour-phase pressure would have to be increased to $592.86 \mathrm{~Pa}$. If the pressure were raised to this value, there would be no net evaporation flux.

distinguishable from the measured pressure for either evaporation or condensation of water. Similar results were found for the evaporation of octane or methylcyclohexane [5].

By making use of Eq. (16) and the measured values of $j_{L V}, T^{V}, T^{L}$, and $R_{0}$, a similar numerical procedure may be followed to calculate the interfacial entropy production at the liquid-vapour interface, $\dot{\sigma}_{L V}$ as a function of $P^{V}$. For the evaporation and the condensation experiments labeled $\mathrm{El}$ and $\mathrm{Cl}$ in Table 1, the calculated values of $\dot{\sigma}_{L V}$ as a function of $P^{V}$ are shown in Figs. 1 and 2, respectively. Note that there is a minimum in the local rate of entropy production and that at the minimum the phase change rate is zero.

The pressures, denoted as $P_{M E P}$ corresponding to the minimum in $\dot{\sigma}_{L V}$ for each experiment are listed in Table 1 where they may be compared with $P_{R}$. Note that for each evaporation experiment, the pressure in the vapour phase when there was a nonzero evaporation rate, is less than the pressure corresponding to a minimum rate of interfacial entropy production. Thus, for given values of $T^{V}, T^{L}$, and $R_{0}$, to bring the system to a condition where there is a minimum rate of interfacial entropy production requires the pressure to be raised. However, raising the vapour-phase pressure to this value has the effect of reducing the evaporation flux to zero. A similar result is found 


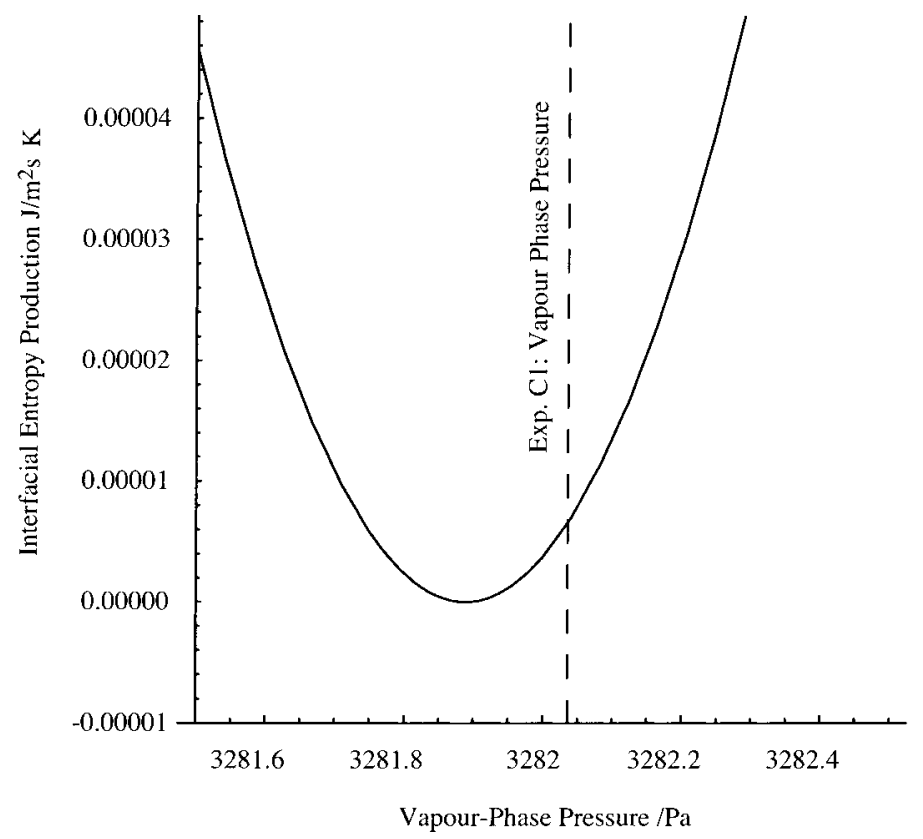

Fig. 2. Rate of interfacial entropy production steady state evaporation as a function of the vapour-phase pressure. The calculations were made for $T^{L}, T^{V}$ and $R_{0}$ of the condensation experiment $\mathrm{C} 1$ (Table 1). The measured condensation flux was $0.315 \mathrm{~g} / \mathrm{m}^{2} \mathrm{~s}$ and the predicted pressure at this condition was $3282.04 \mathrm{~Pa}$. To have the process occurring at the minimum interfacial entropy production rate would require the vapour-phase pressure to be reduced to $3281.89 \mathrm{~Pa}$ which would also reduce the condensation flux to zero.

Table 1. Measured and predicted conditions during evaporation (E1-E4) or condensation (C1-C4) of water from Ref. [1].

\begin{tabular}{|c|c|c|c|c|c|c|c|}
\hline $\begin{array}{l}\text { Exp. } \\
\text { No. }\end{array}$ & $\begin{array}{l}\text { Liquid } \\
\text { Inter- } \\
\text { facial } \\
\text { Temp. } \\
T^{L} \\
{ }^{\circ} \mathrm{C}\end{array}$ & $\begin{array}{l}\text { Vapour } \\
\text { Inter- } \\
\text { facial } \\
\text { Temp. } \\
T^{V} \\
{ }^{\circ} \mathrm{C}\end{array}$ & $\begin{array}{l}\text { Radius } \\
\text { on } \\
\text { Centre- } \\
\text { line, } \\
\mathrm{R}_{0} \\
\mathrm{~mm}\end{array}$ & $\begin{array}{l}\text { Avg. } \\
\text { Evap } \\
\text { Flux } \\
\mathrm{g} / \mathrm{m}^{2} \mathrm{~s}\end{array}$ & $\begin{array}{l}\text { Measured } \\
\text { Vapour- } \\
\text { phase } \\
\text { Pressure } \\
\mathrm{Pa}\end{array}$ & $\begin{array}{l}\text { Vapour- } \\
\text { phase } \\
\text { Pressure } \\
P_{R} \\
\mathrm{~Pa}\end{array}$ & $\begin{array}{l}\text { Vapour- } \\
\text { phase } \\
\text { Pressure } \\
P_{M E P} \\
\mathrm{~Pa}\end{array}$ \\
\hline E1 & $-0.4 \pm 0.05$ & $2.6 \pm 0.05$ & 6.088 & 1.017 & $593 \pm 34$ & 592.4 & 592.86 \\
\hline $\mathrm{C} 1$ & $25.6 \pm 0.05$ & $26.0 \pm 0.05$ & 7.119 & $-0.315 \pm 0.003$ & $3181 \pm 127$ & 3282.04 & 3281.89 \\
\hline E2 & $-0.1 \pm 0.05$ & $2.8 \pm 0.05$ & 6.20 & 0.797 & $639 \pm 34$ & 605.6 & 605.97 \\
\hline $\mathrm{C} 2$ & $19.2 \pm 0.05$ & $19.5 \pm 0.05$ & 4.545 & -0.177 & $2161 \pm 86$ & 2224.5 & 2224.39 \\
\hline E3 & $-0.2 \pm 0.05$ & $2.4 \pm 0.05$ & 6.506 & 0.595 & $616 \pm 34$ & 601.3 & 601.63 \\
\hline $\mathrm{C} 3$ & $12.6 \pm 0.05$ & $13.0 \pm 0.05$ & 6.690 & -0.150 & $1463 \pm 45$ & 1458.8 & 1458.7 \\
\hline E4 & $-0.1 \pm 0.10$ & $2.5 \pm 0.10$ & 6.143 & 0.419 & $629 \pm 34$ & 605.8 & 605.99 \\
\hline $\mathrm{C} 4$ & $6.9 \pm 0.06$ & $7.5 \pm 0.06$ & 5.628 & -0.040 & $959 \pm 32$ & 994.81 & 994.79 \\
\hline
\end{tabular}


in the case of condensation. To stop the steady state condensation process, the pressure in the vapour phase must be reduced (see Fig. 2). This would not bring the system to equilibrium because in principle the temperature discontinuity could be maintained, but it would stop the phase change process, even if the temperature discontinuity were maintained.

\section{Discussion and conclusion}

If the two phase, open system considered were restricted to the case where there was no net molecular transport across the interface, but the discontinuities in the intensive properties were present, the circumstance would correspond to one considered earlier by Prigogine [25]. He showed that provided the Onsager reciprocal relations were valid and the phenomenological coefficients were independent of both the state variables and the generalized forces, such states corresponded to a minimum in the entropy production rate. However, when proved in this way, states corresponding to a minimum rate of entropy production would be limited to those states that were only very slightly displaced from equilibrium (the so called "linear" regime [26]).

If one restricts the open, stationary nonequilibrium system that we have considered to being closed (no net phase change), then as indicated by Eq. (15), $\Delta s_{L V}$ must be zero. When discontinuities in the temperature and chemical potential exist across the phase boundary, this condition could only be met if the property relation given in Eq. (19) were valid. It should be noted that Eq. (19) does not restrict the system to being close to equilibrium. The condition, Eq. (19), was close to being satisfied in each of the water phase-change experiments. Small adjustments in the pressure would have brought the system to the minimum $\dot{\sigma}_{L V}$ state in each case (see Table 1). In these experiments, the largest temperature discontinuity was $3{ }^{\circ} \mathrm{C}$. In others [3], it was as much as $7.8^{\circ} \mathrm{C}$.

The basis for the prediction of $\dot{\sigma}_{L V}$ is statistical rate theory. This theoretical approach is based on the transition probability concept of (conventional) quantum mechanics which was used to calculate each of the unidirectional rates. To apply the transition probability concept, one must say both what phase the molecule is coming from and what phase it is going to. As a result, each of the unidirectional rates is predicted to depend on the thermodynamic state in each of the phases. (See Eqs. (8), (12) and (13)).

It has been previously pointed out that this prediction is in contrast to the predictions obtained from classical kinetic theory [1]. That approach indicates that the unidirectional rate of evaporation only depends on the thermodynamic properties in the liquid phase [e.g., see [24]]. However, the results of classical kinetic theory approach have been recently brought into question by a survey of the values of the evaporation and condensation coefficients reported by different investigators at nominally the same conditions. Merek and Straub [27] report that the values of each coefficient differ from one experiment to another by several orders of magnitude.

A similar circumstance exists with some theories of gas adsorption. For example, transition state theory "supposes" (there is no derivation of the rate expression [28]) 
that the isothermal, unidirectional rate of gas desorption depends only on the conditions in the adsorbed phase. Whereas, the transition probability concept used in SRT indicates that the unidirectional gas desorption rate depends on properties in both the adsorbed phase and in the gas phase. Ideally, the question of the dependence of the unidirectional rate expression should be resolved by experiments. However, to apply either theory requires the expression for the chemical potential of the adsorbed gas. One has been developed [29], and for the CO-Ni(111) system, the parameters appearing in the expression have been determined. This expression for the chemical potential has been used to predict the coverage dependence of the heat of adsorption. When compared with measurements, close agreement was found over a range of coverages [29]. This expression for the chemical potential was also used with SRT to examine beam-dosing adsorption kinetics [10), but certain apparatus constants were not given with the measurements. Although the calculations were in close agreement with the measurements, it was only concluded that the SRT expression for the adsorption rate gave explicitly the coverage dependence and was consistent with the measurements. A more complete examination of SRT and the expression for the chemical potential was possible from experiments that measured the thermal desorption spectra of $\mathrm{CO}$ desorbed from Ni(111). Since in the thermal desorption study, a number of experiments were performed in which only the initial surface concentration of $\mathrm{CO}$ was changed before the heating process was begun, the apparatus constants that were not given could be determined from one experiment, and then the pressure spectra of the other experiments could be predicted. The comparison between the measurements and the predictions give strong support to the SRT expression for adsorption kinetics [11, 30].

Ideally one would use the same expression for the chemical potential of $\mathrm{CO}$ adsorbed on $\mathrm{Ni}(111)$ and determine if transition state theory would give similar results for the thermal desorption spectra of the CO-Ni(111) system. However, the expression for the chemical potential that can: 1) successfully be used to calculate the equilibrium adsorption isotherms and to predict the coverage dependence of the heat of adsorption [29], and 2) used with SRT to predict the thermal desorption spectra of CO-Ni(111) [11] indicates that transition state theory is internally inconsistent [7].

Statistical rate theory also receives experimental support from the kinetics of gas absorption by liquids [19-21]. In this case, SRT predicts that the unidirectional rates of gas absorption depends on the properties in the liquid and the gas phases. As in the case of evaporation, an approximate expression for the equilibrium exchange rate of the absorbing gas may be determined. This expression is in terms of the radius of the gas molecule being absorbed by the liquid phase. The SRT expression was evaluated by determining the value of the molecular radius that gave the best fit of the measured amount of gas absorbed as a function of time. For $\mathrm{O}_{2}$ being absorbed by $\mathrm{H}_{2} \mathrm{O}$ at a temperature of $22.2^{\circ} \mathrm{C}$, the molecular radius of the water molecule was found to be $1.8 \times 10^{-10} \mathrm{~m}$; whereas the average value of this radius determined by three other techniques is $1.78 \times 10^{-10} \mathrm{~m}$ [21]. Thus, gas absorption kinetics, as well as phasechange kinetics and gas adsorption kinetics, indicate that the SRT prediction of the unidirectional rates depending on the condition in both phases has significant experimental support. 


\section{Acknowledgements}

The author gratefully acknowledges the support of the Alexander von HumboldtStiftung, the Canadian Space Agency and the Natural Sciences and Engineering Research Council, Canada and the Lehrstuhl für Strömungsmechanik of the Friedrich-Alexander-Universität, Erlangen-Nuremberg.

\section{References}

[1] Ward, C.A., Stanga, D., Interfacial conditions during evaporation or condensation of water, Phy. Rev. E., 64 (2001), 051509.

[2] McGaughey, A.J.H., Ward, C.A., Temperature discontinuity at the surface of an evaporating droplet, J. Appl. Phys., 91 (2002), 6406.

[3] Fang, G., Ward, C.A., Temperature measured close to the interface of an evaporating liquid, Phy. Rev. E., 59 (1999), 417.

[4] Ward, C.A., Fang, G., Expression for predicting liquid evaporation flux: Statistical rate theory approach, Physical Review E., 59 (1999), 429.

[5] Fang, G., Ward, C.A., Examination of the statistical rate theory expression for liquid evaporation rates, Phy. Rev. E., 59 (1999), 441.

[6] Ward, C.A., Findlay, R.D., Rizk, M., Statistical rate theory of interfacial transport, I. Theoretical development, J. Chem. Phys., 76 (1982), 5599.

[7] Ward, C.A., Elliott, J.A.W., Material properties controlling adsorption kinetics and temperature programmed desorption spectra, Appl. Surf. Sci., in press.

[8] Ward, C.A., Findlay, R.D., Statistical rate theory of interfacial transport. III. Predicted rate of non Non-dissociative adsorption, J. Chem. Phys., 76 (1982), 5615.

[9] Findlay, R.D., Ward, C.A., Statistical rate theory of interfacial transport. IV. Predicted rate of dissociative adsorption, J. Chem. Phys., 76 (1982), 5624.

[10] Elliott, J.A.W., Ward, C.A., The statistical rate theory descriptioin of beam-dosing adsorption kinetics, J. Chem. Phys., 106 (1997), 5667.

[11] Elliott, J.A.W., Ward, C.A., Temperature programmed desorption kinetics: A statistical rate theory approach, J. Chem. Phys., 106 (1997), 5667.

[12] Rudzinski, W., Panczyk, T., Kinetics of isothermal adsorption on energetically heterogeneous solid surfaces: A new theoretical description based on the statistical rate theory of interfacial transport, J. Phys. Chem. B., 104 (2000), 9149.

[13] Rudzinski, W., Borowiecki, T., Panczyk, T., Dominko, A., A quantitative approach to calculating the energetic heterogeneity of solid surfaces from an analysis of TPD peaks: Comparison of the results obtained using the absolute rate theory and the statistical rate theory of interfacial transport, J. Phys. Chem. B., 104 (2000), 1984.

[14] Rudzinski, W., Borowiecki, T., Panczyk, T., Dominko, A., Theory of thermodesorption from energetically heterogeneous surfaces: Combined effects of surface heterogeneity, readsorption, and interactions between the adsorbed molecules, Langmuir, 16 (2000), 8037.

[15] Torri, M., Elliott, J.A.W., A statistical rate theory description of CO diffusion on a stepped Pt(111) Surface, J. Chem, Phys., 111 (1999), 1686.

[16] Elliott, J.A.W., Elmoazzan, H.Y., McGann, L., A method whereby onsager coefficients may be evaluated, J. Chem. Phys., 113 (2000), 6573.

[17] Ward, C.A., Effect of concentration on the rate of chemical reactions, J. Chem. Phys., 79 (1983), 5605.

[18] Dejmek, M., Ward., C.A., Study of interface concentraction during crystal growth of dissolution, J. Chem. Phys., 108 (1998), 8698.

[19] Ward, C.A., Rizk, M., Tucker, A.S., Statistical rate theory of interfacial transport. II. Rate of Isothermal bubble evolution in a liquid-gas solution, J. Chem. Phys., 76 (1982), 5606. 
[20] Ward, C.A., Tikuisis, P., Tucker, A.S., Bubble evolution in solutions with gas concentrations near the saturation value, J. Colliod Interface Sci., 113 (1986), 388.

[21] Tikuisis, P., Ward, C.A., Rate of Gas Absorption by Liquids and Surface Resistance, in Transport Processes in Bubbles, Drops and Particles, p. 114, Eds. Chhabra, R.P., De Kee, D., Hemisphere, New York, 1992.

[22] Prigogine, I., Laws of nature, probabilty and time symmetry breaking, Physica A, 263 (1999), 528.

[23] Herzberg, G., Molecular Spectra and Molecular Structure, Vol. 2, p. 281, Van Nostrand, New Jersey, 1964.

[24] Knacke, O., Stranski, I.N., The mechanism of evaporation, Progr. Metal Phys., 6(1965), 181.

[25] Prigogine, I., Introduction to Thermodynamics of Irreversible Processes, 3rd Ed., p. 75, Interscience, New York, 1995.

[26] Nicolis, G. Introductory remarks: Thermodynamics today, Physica A, 213 (1995), 1.

[27] Marek, R., Straub, J., Analysis of the evaporation coefficient and the condensation coefficient of water, Int. J. Heat and Mass Transfer, 44 (2001), 39.

[28] Zhdanov, V.P., Adsorption-desorption kinetics and chemical potential of adsorbed and gasphase particles, J. Chem. Phys., 114 (2001), 4746.

[29] Elliott, J.A.W., Ward, C.A., Chemical potential of adsorbed molecules from a quantum statistical formulation, Langmuir, 13 (1997), 951.

[30] It is hard to understand the comment of [28] to SRT in part because of the notation in the article. In a self-contradictory paragraph that he first says "to prevent confusion" the notation will follow that of Ref. [6], but then, in the same paragraph, says on certain key concepts "our terminology" is "different". Also the view is expressed in [28] that molecular adsorption or desorption are each "multistep" processes, and that the transition probability concept can not be used to predict the probability of such a process in which a molecule makes a transition form one phase to another because the "direct transition from the adsorbed state (near the bottom of the potential well) to the gas phase is practically impossible" because the molecule can not get the required energy from the substrate. However, in the SRT approach molecules in an adsorbed or any other phase are allowed to have an energy distribution as predicted for a canonical ensemble [4]. Some of the molecules would be near the bottom of the energy distribution, but some would be near the top. In the SRT approach one does not predict which molecule makes a transition only that a molecule from one phase makes a transition to another phase. The only restriction on the transition probability concept is that whatever the interaction involved in a particular transition, it must take place in a limited period of time, $\Delta t$. This limitation has been investigated for evaporation, and it does not appear to restrict the application of the transition probability concept. For example, for evaporation it was found that $10^{-4} \mathrm{~s} \geq \Delta t \leq 8 \times 10^{-14} \mathrm{~s}[7]$.

Reference [28] also claims that a sum over states as used in the SRT development of the rate expression can not be performed, and says this can be "proved by reading a paper". How reading a paper could possibly prove such a claim is not clear. He also misunderstands the reason for referencing Pauli and Tolman in Ref. [6]. These earlier authors were referenced because of the similarity of the mathematical techniques used by them and those used in Ref. [6]. The justification of the SRT approach at the time was the comparison of the predictions obtained from SRT with experiments. Three papers, [8], [9] and [19] were submitted simultaneously with Ref. [1]. Each compared predictions with experimental data from a different field. More rigorous comparisons have been made since.

It is claimed in [28] that SRT predicts the unidirectional desorption rate is inversely proportional to the pressure, but this claim is also false. Statistical rate theory indicates that in an isolated system the desorption rate is proportional to the ratio $P_{e} / P$. The value of this ratio depends on the constraints acting on the system. For example, if the process is taking place in a large system where the adsorption does not significantly change the pressure, this ratio would be unity [10]. 
Paper received: 2002-03-12

Paper accepted: 2002-03-27

Prof. Charles A. Ward

Dept. of Mechanical and Industrial Engineering

University of Toronto

5 King's College Road

Toronto, M5S 3G8

Canada

E-mail:ward@mic.utoronto.ca

present address:

Lehrstuhl für Strömungsmechanik

Technische Fakultät

Friedrich-Alexander-Universität

Erlangen-Nürnberg

Cauerstraße 4

91058 Erlangen

Germany 